\title{
A REVIEW ON ENHANCING ABIOTIC STRESS TOLERANCE IN RICE AND WHEAT GENOTYPES
}

\author{
JHILICK BANERJEE* \\ Research Scholar at Department of Plant Breeding and Genetics, College Of Agriculture, JNKVV, Jabalpur,
}

Madhya Pradesh, India

\begin{abstract}
Abiotic stress tolerance is complex but as the technologies are improving, the components associated with abiotic stress tolerance can be accessed easily. Abiotic stresses like drought, heat, salinity have a devastating effect on crop yield. Rice and wheat being the most popular staple crop are highly affected by abiotic stress during their growth stages. This reduces their grain yield which directly affects food security. Hence it becomes necessary to develop cultivars that can withstand the problem of climate change without any compromise on yield. The genetic mechanisms that control abiotic stress tolerance are still yet to be understood. The abiotic stress tolerance traits are polygenic in nature and the majority of them are directed by Quantitative trait loci (QTLs) that are present on different chromosomes. These QTLs responsible for abiotic stress tolerance are identified using molecular markers and are then used in marker assisted selection to identify tolerant genotypes. Abiotic stress tolerance can also be produced by introgressing desirable traits into the cultivar by exploiting the existing germplasm with the help of conventional breeding methods. This review highlights the abiotic stresses of particularly the two most important crops i.e rice and wheat also the different methods employed to enhance tolerance in genotypes. Also, it highlights the role of various biochemical compounds found within the crops that provide tolerance to abiotic stress.
\end{abstract}

KEYWORDS: Polygenic, Quantitative Trait Loci, Marker Assisted Selection \& Introgressing

Received: Jul 04, 2021; Accepted: Jul 24, 2021; Published: Aug 06, 2021; Paper Id.: IJASRDEC202118

\section{INTRODUCTION}

The decline in the availability of natural resources due to the spontaneous increase in the global population has hit the alarm bell of increase in the demand for food, accompanying this is the issue of climate change which affects the agriculture sector directly. The effect of climate change on crops is extremely disheartening as a reduction in crop yields directly affects food and nutritional security. Therefore it is desirable to produce abiotic stress tolerant crops that are adaptive to extreme environments and climate. These tolerant crops provide higher and stable yields even during the harsh effects of climate variability. Crops endure a lot of abiotic stresses such as temperature, salinity, drought, mineral toxicity etc. These factors play a primary role in reducing crop yields. It has been estimated that approximately more than $50 \%$ of global yield loss for major crops are because of abiotic stresses. (Shao et al., 2009; Ahuja et al., 2010; Lobell et al., 2011). Stress is also defined as an environmental condition that suppresses genetic expression. Climate variability is known to be one of the biggest sources of abiotic stress. However, plants do have multiple signalling pathways that are activated based on the type of stress. These defence systems used by plants are triggered immediately after the stress is recognized. For example, the generation of reactive oxygen species (ROS), the ion channels are stimulated by abscisic acid, salicylic acid, ethylene and jasmonic acid. These defence responses help in reducing the loss caused by that particular stress this forms the basis 
of crop abiotic tolerance. (Younis et al.2020) Stress cause the plant to divert from the normal life cycles. These stress affected plants exhibit three common response phase first is the initiation phase followed by the activation phase this is the phase where the exact defence system respond lastly the exhaustion phase which exhibits the loss due to the stress. Out of all the abiotic stresses that affect plants salinity is the one that factor that hinders plant growth and productivity as it affects water accumulation and retention thus affecting iron homeostasis. On the other hand, drought is when there is a lack of availability of water which is also associated with excessive evapotranspiration. Drought causes a reduction in survival and growth of plants. This drought stress usually occurs when there is an imbalance between the water uptake and losses through transpiration. Mineral toxicity is another example of abiotic stress where there is an excess of a mineral which again affects the homeostasis management of ions within the plants. The temperature stress is variable depending upon the geographical location if the temperature is low then the stress is categorized as chilling stress (temperature is above 0 degree Celsius) and freezing stress (temperature is below 0 degree Celsius). When the temperature exceeds the optimum temperature of plants then it is called heat stress which adversely affects the crops by retarding their growth and productivity.

The abiotic stresses in the agriculture sector have proved to be of great threat to food security and the saddest part is that these stresses are intensified by the effects of global warming and climate change. Therefore these adverse effects can be avoided by the production of stress tolerant crops. However, there have been a number of genes that have been identified that respond to abiotic stresses. Although with the increasing trends in technology in the field of genomics and biotechnology allow enough exposure to crops to stresses so that they become tolerant to that particular stress. For any breeding strategy and transgenic approach to work, it is very necessary to identify genes thus it is a prerequisite for the development of stress tolerant crops. Recent strategies work towards engineering a group of regulatory genes which affects the controlling of many stress responsive genes. Breeding of abiotic stress tolerant crops with improved yields and enhanced tolerance against multiple abiotic stresses is an effective strategy as well as a challenge for agriculture sector.

\subsection{Impact of Abiotic Stress on Rice and Wheat}

Around $91 \%$ of the world agriculture area is affected by stress and more than $50 \%$ of crop production is under loss because of such stresses. These abiotic stresses retard the farm economic growth as there is reduction in income and lifestyle of farmers. It is evident from Daryanto et al. (2016) that lowering of $40 \%$ in water retards the growth of about $40 \%$ in maize and similarly $21 \%$ in wheat. As discussed above that plants generate ROS but certain abiotic stress stimulate the excessive production of ROS which is toxic and destroys the plant biomolecules and therefore affect plant growth adversely. Zlatev and Lidon.(2012)

Abiotic stresses also have an impact on the biochemical and physiological pathways of crops. Many phytoharmones, antioxidants and various plant enzymes get activated in response to stress these on activation produce certain stress based metabolites to prevent the crops from cellular damage.

Among the major cereals, rice is the most vulnerable crop to drought stress (Showler, 2016) because it directly affects the phenological traits and prolongs the crop duration. (Shao et al. 2008) The effect of drought starts from germination stage and extends up to the time of harvest. It was studied by Kaya et al. 2006 that these dry spells affect germination negatively, there is a decrease in the germination rate however the concentration of polyethylene glycol expands, this causes a reduction in length of hypocotyl along with decrease in biomass. Cell development is also retarded due to poor turgor pressure. Drought stress leads to abnormal mitosis and cell enlargement which lead to stunted growth 
and poor development of yield traits in rice. (Hussain et al. 2008)

Salinity stress in rice accumulates $\mathrm{Na}^{+}$and $\mathrm{Cl}^{-}$which harms the arrangement of roots. $\mathrm{Cl}^{-}$accumulation is evident by broad leaf cutting edge and $\mathrm{Na}^{+}$accumulation results in leaf mottling and rolling. A large amount of $\mathrm{NaCl}$ enters the plant via transpiration which causes extreme damage to the leaves leads to decline in the rate of development. However, rice crops manage salt stress by mechanisms like decreased salt uptake, stomata closure, increased water utilisation efficiency, regulation of antioxidant system etc.

It is seen that heat stress has a negative impact on different growth stages of rice. Increase in the mean temperature from the optimum temperature at important growth stages leads to reduction in grain yield. Most sensitive stages are the vegetative and reproductive stage where a little increase in day temperature will lead to early generation of botanical buds and opening of blooms at conceptive stage. (Guilioni et al.1997) The conceptive stage is more sensitive than the vegetative stage. (Peng et al. 2004)

Wheat is one of the necessary cereal crops having a good amount of calories and proteins for more than $80 \%$ population. With the increasing issue of global warming as well as climate change the impact of heat stress is gaining attention with respect to wheat production. Heat and drought are the most common abiotic stresses found in wheat. It was estimated that increase in temperature by just $1^{\circ} \mathrm{C}$ causes a decrease in global wheat production by almost $6 \%$. (Asseng et al.2011)

Heat stress in wheat results in the deactivation of Rubisco enzyme, reduction in chlorophyll content, decrease in photosynthetic capacity, reduction in assimilation rate of translocation, premature leaf senescence, decrease in the duration of grain filling, less number of grains, poor seed germination and ultimately loss in yields. Heat stress also affects the biochemical pathways of wheat by affecting the starch and protein content in wheat. As in the case of rice heat stress produces ROS in wheat which leads to change in membrane stability and other side effects. Wheat has evolved a number of tolerance mechanisms to heat stress which includes the production of heat shock proteins in response to heat stress that regulates protein folding and unfolding. The ROS concentration is detoxified by the antioxidant derived defence system. Traits like stay green, canopy temperature and chlorophyll fluorescence are associated with heat tolerance in wheat.

Drought stress can have numerous effects involving morphological, physiological and biochemical effects. It was seen by Lonbani and Arzani that the length and area of flag leaf in wheat increased in drought stress whereas the width did not change. Leaf area is reduced during drought stress which hinders the rate of photosynthesis. Therefore the morphological effects include the decrease in the number of leaves per plant, shrinkage in the leaf area and size also there is retardation in the longevity of leaves. The translocation of metabolites is highly affected due to water stress as the sink strength is reduced during the early stages of grain filling which results in fewer endosperm cells and loss of metabolic activity.

Thus it can be concluded that there are variable abiotic stresses viz. heat, drought, salinity, cold, mineral toxicity etc that have a negative impact on crops at different stages starting from germination to harvest. By looking at the adverse effects that abiotic stress has on crop morphology, physiology and biochemical pathways it is evident that there is a need for promising solutions to face abiotic stress which can be done by developing new cultivars that show tolerance towards these stresses and yield is maintained. 


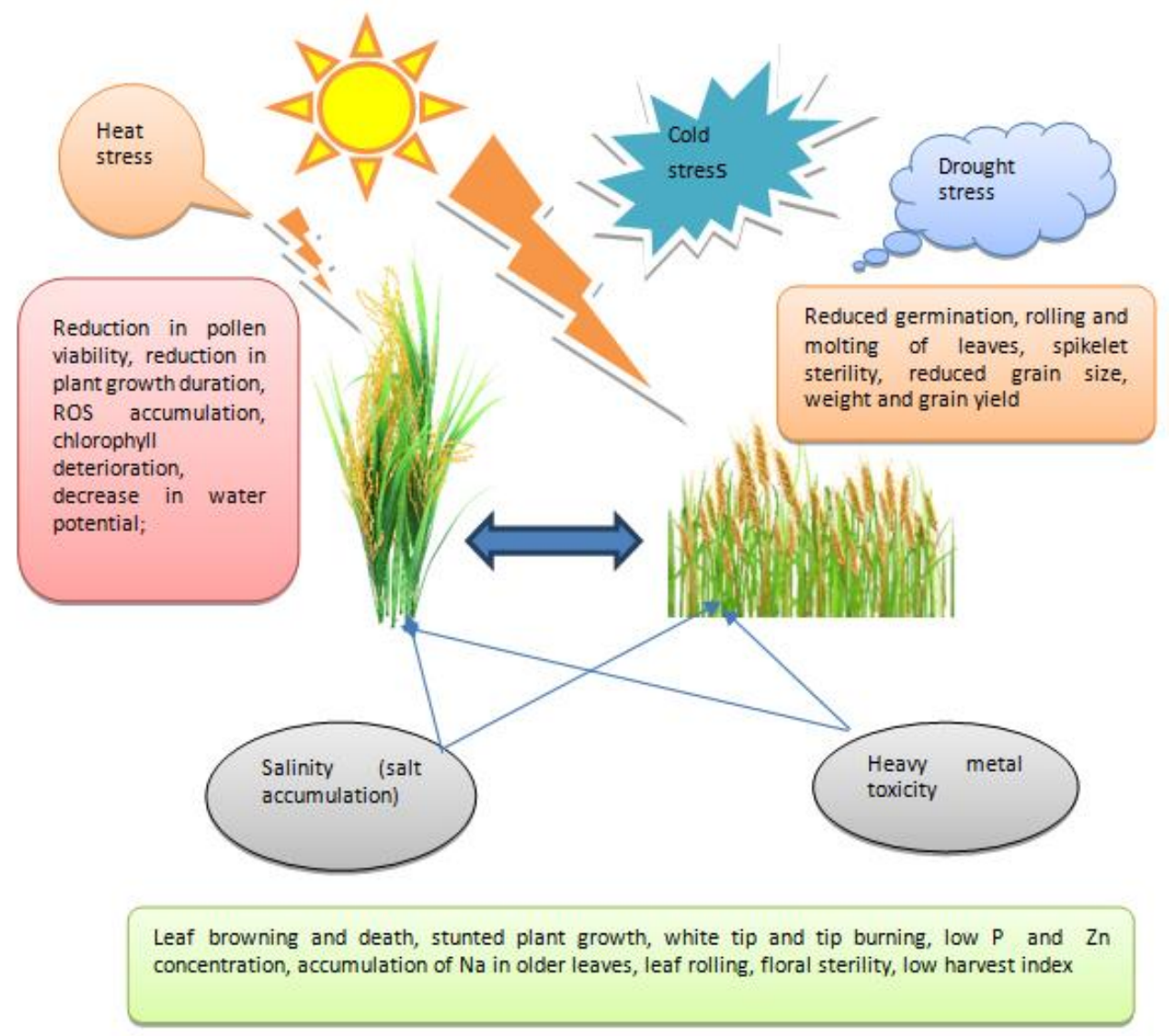

Figure 1: The Impact of Various Abiotic Stress on Rice and Wheat.

\section{EXPLOITING GENETIC DIVERSITY FOR MULTIPLE STRESS TOLERANCE IN RICE}

Superior rice varieties tolerant against multiple stress was developed by introgression breeding by Ali et al.2017. It was done with the help of backcross populations where eight $\mathrm{BC}_{1}$ populations were derived from an adaptable recipient along with eight donors with phenotypic selection. 496 introgressed lines were developed which showed higher yield under adverse conditions of drought and salt. It was reported that 6 novel varieties were released in the Philippines and Pakistan and majority of them are under multilocation trials. This breeding procedure has certain characteristics that were found using marker facilitated genetic characterization. The inrogression frequency was variable in separate genomic regions which was due to strong selection of traits, apart from this it was also seen that the $\mathrm{BC}$ progenies exhibited less heterozygosity for drought and salinity tolerance. The introgression along with phenotypic selection improves multiple abiotic stress and also increases homozygosity in early generations. This breeding method is ideal for use in small breeding programs to develop high yielding genotypes coupled with abiotic stress tolerance in rice.

Recently it was seen that recurrent selection could be applied in rice breeding because of a new male sterile line was found named 'Jiabuyu' that was controlled by a single dominant gene, this proved to be a milestone in rice breeding as it paved a way for recurrent selection. Two different recurrent selection populations that were compatible for crossing with the dominant sterile line were developed. Out of this one of them utilized 31 abiotic stress tolerant introgressed lines that had common recipients and the other one used 25 restorer lines for hybrid breeding. After the $2^{\text {nd }}$ cycle of recurrent selection the seeds from the male fertile lines were screened in 3 conditions viz. normal, irrigated, drought and salt stress. 
The screening results reported 12 drought tolerant and 12 lines that exhibited salt tolerance. The above study by Pang et al. 2017 was an ideal research of utilizing recurrent selection by a dominant male sterile line for the improvement of rice.

\subsection{Exploiting Genetic Diversity for Multiple Stress Tolerance in Wheat}

Drought stress is considered as a huge bottleneck in wheat improvement. The usage of wild relative viz. Aegilops tauschii (DD) is regarded to be a reliable source for creating genetic variability. In a study by Faheem et al. 2015 they assessed the D-genome derived genetic diversity in drought tolerant germplasm with the help of SSR markers. It was found out that 178 alleles occupied 49 loci at an average rate of 3.49 alleles per locus. The genetic diversity that was studied using these SSR markers reported that lowest was $4 \%$ and highest was $67 \%$ with average of $56.2 \%$ per marker. There number of SSR marker which reported PIC (Polymorphism information content) were 23. This value was for more than 50\% while on an average the PIC values for all markers ranged from 8-63.2\% having an average of $48.1 \%$. Thus this study concluded that utilizing this germplasm can be used for improving wheat cultivars for drought tolerance in rainfed environments.

In order to assess the outcomes of introgressing synthetic hexaploid wheat diversity into the winter wheat of the U.S. Great Plains Becker. (2014) came up with a technique. It is a feasible technique to improve the yield and drought stress tolerance in wheat by introgressing new alleles from ancestral species. A group of six synthetic hexaploid wheat lines were identified from a collection of 412 lines for developing drought stress tolerance in winter wheat.

Heat stress in wheat and the variability in rainfall accompanying dry spells directly affects grain yield which indirectly affects food security globally. Therefore the most important source for isolating desirable genes is by utilizing the existing genetic resources for enhancing future gain in the yield ability of wheat under adverse stress conditions. 44 wheat landraces were characterised for heat tolerance and yield potential in a study conducted by Gulnaz et al. 2019. The heat tolerance value was found to show positive significant correlation with grain yield continuously for almost three years. The three prominent heat tolerant lines identified can be used as genetic resources for addressing the problem of heat stress by introgressing heat tolerance trait into agronomic ally high yielding wheat cultivars.

Thus by reviewing the above studies we can conclude that conventional breeding strategies can also be employed to existing genetic resources to produce abiotic stress tolerant genotypes. There is enormous genetic diversity that exists for the dissection of valuable traits regarding stress tolerance these traits can be exploited and introgressed into high yielding cultivars.

\section{MARKER ASSISTED SELECTION FOR HEAT TOLERANCE IN WHEAT}

Conventional phenotypic selection is a traditional method for developing stress tolerant cultivars however the success rate of conventional breeding as compared to new marker assisted breeding is less. Apart from this the phenotypic selection method is a bit difficult. These stress tolerant traits are polygenic also accompanied with complex pattern of inheritance. Due to the presence of majority of QTLs for a complex trait like this marker assisted selection becomes an effective method for screening tolerant genotypes. In a study conducted by Sadat et al. 2013 where they screened out 25 bread wheat genotypes for heat stress. In this study around fourteen SSR markers were used that were associated with yield attributing traits like grain filling duration, HSI (Heat susceptibility index) etc. were evaluated. A dendrogram was constructed using the UPGMA which grouped the genotypes into 3 clusters. This molecular screening along with previous results of field experiments indicated that the markers could screen bread wheat for heat tolerance. 
A similar study was performed by Gupta et al. 2015 where microsatellite markers were employed for the genetic analysis of the trait terminal heat tolerance using F2 population of wheat. The DNA from two parents where one was derived from normal and the other from late sown conditions, were amplified by 200 SSRs. Out of which only 22 SSRs exhibited polymorphism and an average of 1.45 alleles. It was seen that 13 QTLs for A genome 5 QTLs for B genome and 9 QTLs for D genomes were identified. Out of these 9 QTLs were resolved after composite interval mapping and all these identified 9 QTLs were located on three different chromosomes. These markers will be efficient in saving time and energy for producing segregating generations for estimating terminal heat tolerance.

\section{QTL MAPPING FOR DROUGHT TOLERANCE IN RICE}

The development of drought tolerant rice cultivars will help in mitigating yield losses due to drought stress in rice cultivars. The genetic, morphological and molecular analysis of rice germplasm suggested scope of improvement as it revealed a good amount of genetic variation for drought tolerance within the rice gene pool. With the help of information about the rice genome sequence availability of molecular markers and cost effective genotyping techniques makes it feasible to apply genomics and markers assisted technologies to improve grain yield under drought stress.

Major QTLs have been identified that are associated with grain yield under drought. Also, QTLs linked to different drought associated traits were mapped on rice chromosome 1,2,3,9 and 12 in an experiment conducted by Vikram et al. 2012. And till now research is undergoing to identify genes that underlay these QTL. Direct selection for yield and its attributing traits under adverse conditions of drought is an important criteria while enhancing drought tolerance in rice.

There has been a considerable amount of progress made in mapping of QTLs for drought tolerance in rice and a lot of them have been used in MAB. In an experiment conducted by Prince et al. 2015 recombinant inbred lines and two of indica cultivars that were well adapted to rainfed environmental condition were evaluated in two seasons under managed stress condition and in a rainfed drought stress environment respectively.

Another milestone in QTL mapping for drought tolerance in rice was carried out by Sabar et al. 2019 where QTLs related to drought resistance and yield parameter in a cross between IR55419-04 and Super basmati. The mapping population consisted of an $\mathrm{F}_{2}$ population of 418 individuals. Two extreme lines i.e one with tolerance and the other highly sensitive were selected. 13 microsatellite markers were run across the genome to identify the polymorphism between these two parents. 21 QTLs got discovered after composite QTL mapping. Those QTLs that were linked to drought tolerance were located on chromosome 1 in the marker interval between RM520-RM143 and RM168-RM520. This region also contained a QTL QTWU3.1 which was responsible for water uptake. Another QTL responsible for root morphological trait was found in chromosome 3. These QTLs can be used for marker assisted breeding to produce drought tolerant rice cultivars and genotyping of these QTL will help us to describe the relationship between these QTL and phenotypic traits.

Drought is one of the most limiting factor when it comes to rice production however the genetic mechanism of drought tolerance is still unknown. To study this a study was conducted by Kim et al. 2017 where they screened out 218 rice genotypes to select drought tolerant varieties. Traits like visual drought tolerance (VDT) and relative water content (RWC) were recorded in a double haploid population to identify QTLs. Three QTLs were found for VDT were the reason for $41.8 \%$ of the phenotypic variance. The drought tolerance associated QTLs viz. Qvdt2 and Qvdt6 had no effect on tiller formation but other QTL viz Qvdt11 increased the tiller number. It was also observed that when the QTLs Qvdt2 and Qvdt11 are combined it exhibited increased tiller formation. Therefore those DH lines with both these QTLs showed stable 
tiller formation. This concluded that Qvdt11 is important for drought tolerance in rice.

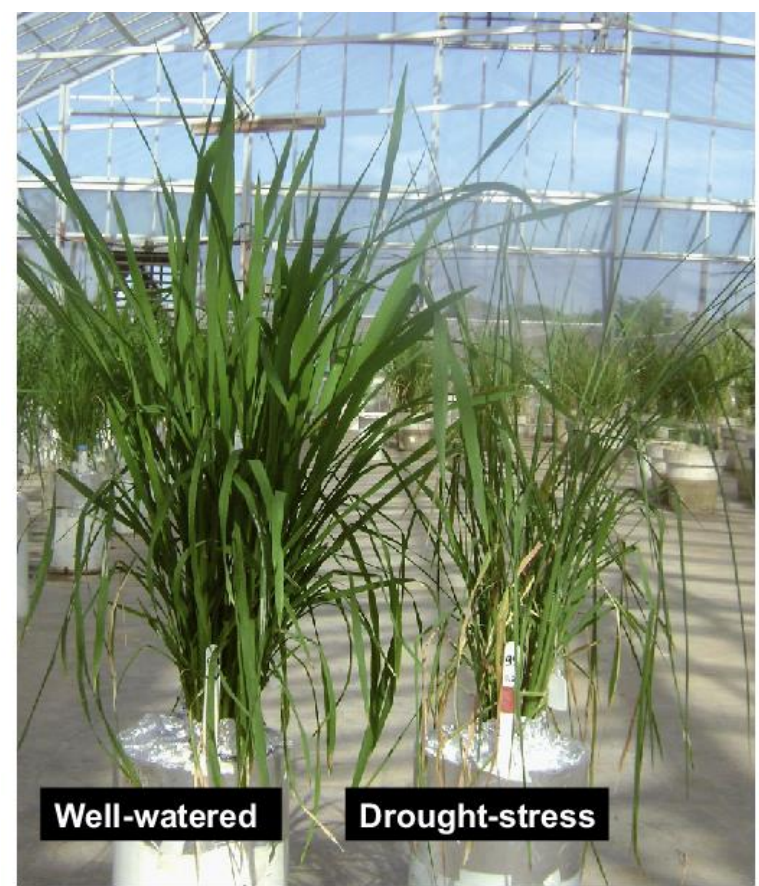

Figure 1: Source -Farooq et al.2008.

\section{QTL MAPPING FOR HEAT TOLERANCE IN WHEAT}

As already discussed above abiotic stress during various vegetative and reproductive stages of a crop can prove detrimental to plant growth. Similarly, higher temperature at seedling stage of early sown winter wheat proves to be detrimental to the productivity of wheat worldwide. To address the problem of seedling heat tolerance Maulana et al.2018 identified QTLs by using SNP markers that were related with seedling heat tolerance. This could only be possible by the use of genome wide association study (GWAS). 200 miscellaneous emblematic lines of hard red winter wheat were used and genotyping with iSelect 90K SNP array reported that these QTLs were located on chromosomes that contain QTLs responsible for heat tolerance during flowering stage. Therefore the inferences that can be made from these results are that these QTLs are effective for heat tolerance from seedling to reproductive stage. However, there were few exceptions of some QTLs that corresponded to heat tolerance only during the seedling stage. This study was one of its kind as it was for the first time that GWAS was employed to map QTLs related to seedling heat tolerance. The SNP markers selected can be further used for Marker assisted selection for heat tolerance in wheat breeding.

A study performed by Lu et al.2020 where they used near isogenic line which differed at only one locus. These NILS were characterised from two populations to target a major 7AL QTL that controlled heat tolerance. Evaluating the chlorophyll content using SPAD values and molecular screening for yield attributing traits paved the way for eight wheat NILs that were contrasting with respect to heat stress responses. Five SNP markers were identified after genotyping the NILs that were associated with heat tolerance from these seven candidate genes which were related to the SNPs were identified as valuable in important processes and metabolic pathways related to heat tolerance in wheat. 


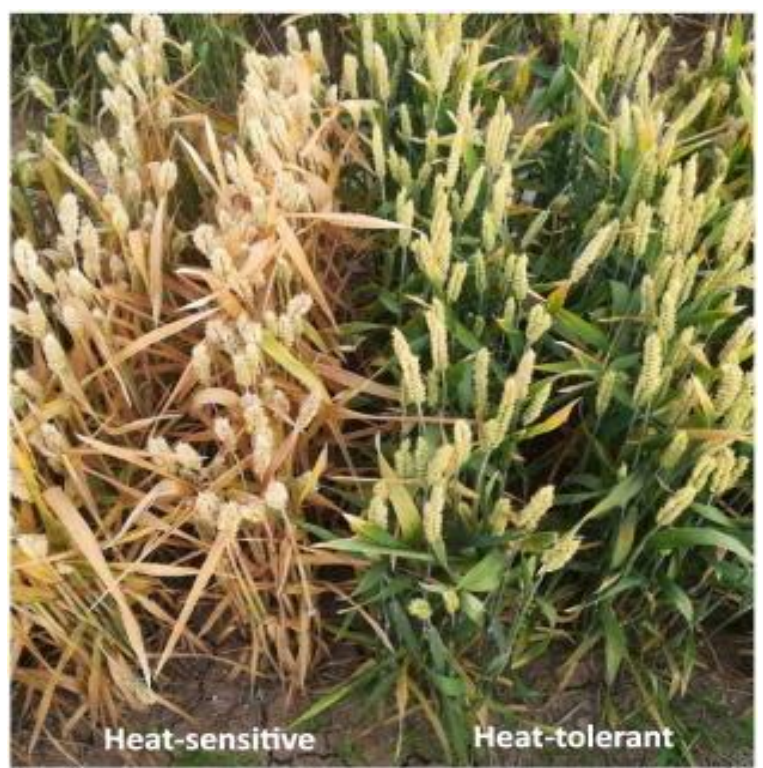

Figure 3: Source-Ni et al. 2018.
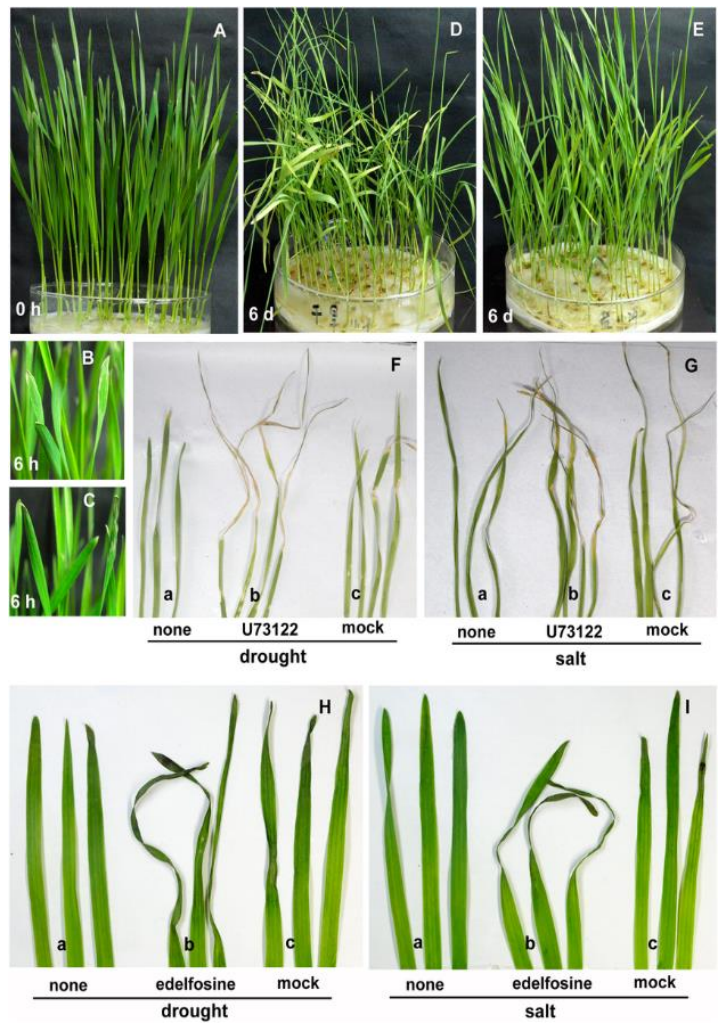

Figure 2: Effect of Drought and Salt Stress on Wheat Seedlings; Source- Zhang et al. 2014.

\section{ROLE OF BIOCHEMICAL COMPOUNDS IN ABIOTIC STRESS TOLERANCE IN RICE AND WHEAT}

Plant hormones or phytohormones are responsible for the control of developmental processes and signalling pathways in crops under stress conditions. The most common phytohormones that eradicate the negative effects of stress are jasmonic acid and salicylic acid. These two phytohormones have shown tremendous potential in abiotic stress tolerance in plants. 
Jasmonates are known to regulate cellular responses from seed germination to fruit ripening and senescence. Jasmonic acid also plays a role in plant responses to stress like drought, salt and heat. Similarly, salicylic acid (SA) also plays an important role in regulating physiological and molecular mechanisms to adjust the crop in adverse environmental conditions. Recently in an experiment conducted by Ilyas et al.2017 to study the effect of salicylic acid and jasmonic acid on wheat during drought stress. The seeds were primed by JA and SA. Usually, drought decreases germination by $26 \%$ but in this case, an increase of $27 \%$ and $21 \%$ was seen in germination after application of JA and SA respectively. The application of JA and SA produced long shoots under drought conditions. These biochemicals lead to an increase in the proline and soluble sugar content also. These results suggest that Jasmonic and Salicylic acid have a great potential to improve the growth of wheat under drought conditions.

Another potential osmoprotectant that protects the crops during abiotic stress is Glycinebetaine (GB). In a study conducted by Farooq et al.2008, the role of glycine betaine for the improvement of drought tolerance in rice was evaluated in a cultivar. GB was applied on both seed and foliage. Drought conditions were properly maintained at 50\% field capacity. The application of GB improved the growth of rice under both drought conditions as well as watered conditions. Drought tolerance in rice was characterized as maintenance of the tissue water potential under water scarcity, this improved the cohesion of cellular membranes and thus the plant was able to photosynthesize more. Foliar treatments of GB gave better results compared to seed treatment.

Glycinebetaine was found protective towards the plasma membrane of wheat at the time of cold stress. In an experiment by Zhang et al.2010 where they used the wild cultivar and a transgenic cultivar which carried the BADH gene that was isolated from Atriplex hortensis. It was seen that during cold stress over accumulation of glycine betaine in the transgenic cultivars provided them with better membrane integrity and the $\mathrm{H}^{+}$ATPase activity was also found to be higher. The results also showed that the production of ROS in the transgenic cultivars were lower. Therefore Glycinebetaine enhanced the cold stress tolerance in wheat.

\section{CONCLUSIONS}

Therefore it was evident from the above literature that abiotic stress has a detrimental effect on the grain yield of the two most important staple crops worldwide. Abiotic stress including drought, salinity and heat are the most common form of stress that affect rice and wheat cultivars during different developmental stages from germination to harvest. The success rate of breeding climate resilient genotypes that can withstand abiotic stress depends upon the holistic efforts of multiple research domains involving plant physiology, plant molecular biology, genetics, biochemistry and plant pathology. It was also seen that with the upcoming current advances in research in the area of genomics assisted breeding and molecular biology the identification and introgression of desirable traits associated with tolerance have become easier but that does not mean that conventional breeding techniques have become outdated, in fact with the help of conventional breeding also we can get promising tolerant genotypes that could be a valuable genic resource for future research. More importance should be given to develop cultivars showing tolerance towards multiple stress and not just one so that they are capable of tolerating the variable consequences of climate change without any reduction in yields. However, the evaluation of the transgenic cultivar developed for tolerance has to be exposed to various stress conditions for studying the physiological effects of the inserted gene is still a cumbersome process. Using genome editing technologies like gene silencing or microRNA mediated silencing of genes are also under process to overcome this problem of abiotic stress tolerance. Thus it can be concluded that abiotic stress tolerance should be given importance in every breeding programme to develop tolerant 
cultivars that will not collapse during adverse conditions and thus it will be possible to procure food for such an increasing population.

\section{REFERENCES}

1. Ahuja, I., de Vos, R. C., Bones, A. M., and Hall, R. D. (2010). Plant molecular stress responses face climate change. Trends Plant Sci. 15, 664-674.

2. Akram, R., Fahad, S., Masood, N., Rasool, A., Ijaz, M., Ihsan, M. Z., ... \& Nasim, W. (2019). Plant growth and morphological changes in rice under abiotic stress. In Advances in Rice research for abiotic stress tolerance (pp. 69-85). Woodhead Publishing.

3. Ali, J., Xu, J. L., Gao, Y. M., Ma, X. F., Meng, L. J., Wang, Y., .. \& Li, Z. K. (2017). Harnessing the hidden genetic diversity for improving multiple abiotic stress tolerance in rice (Oryza sativa L.). PLoS One, 12(3), e0172515.

4. Asseng, Senthold, Foster I, Turner NC. The impact of temperature variability on wheat yields. Global Change Biol. 2011; 17:997-1012.

5. Becker, S. R. (2014). Exploiting drought tolerance traits and genetic diversity of synthetic hexaploid wheat in winter wheat breeding (Doctoral dissertation, Colorado State University).

6. Daryanto, S.; Wang, L.; Jacinthe, P.A. Global synthesis of drought effects on maize and wheat production. PLoS ONE 2016, 11, e0156362.

7. Faheem, M., Mahmood, T., Shabbir, G., Akhtar, N., Kazi, A. G., \& Mujeeb-Kazi, A. (2015). Assessment of D-genome based genetic diversity in drought tolerant wheat germplasm. International Journal of Agriculture and Biology, 17(4).

8. Farooq, M., Basra, S. M. A., Wahid, A., Cheema, Z. A., Cheema, M. A., \& Khaliq, A. (2008). Physiological role of exogenously applied glycinebetaine to improve drought tolerance in fine grain aromatic rice (Oryza sativa L.). Journal of Agronomy and Crop Science, 194(5), 325-333.

9. Gulnaz, Saima, Muhammad Zulkiffal, Muhammad Sajjad, Javed Ahmed, Muhammad Musa, Muhammad Abdullah, Aneela Ahsan, and A. Rehman.2019. "Identifying Pakistani wheat landraces as genetic resources for yield potential, heat tolerance and rust resistance." International Journal of Agriculture and Biology 21: 520-526.

10. Gupta, M., Chawla, V., Garg, P., Yadav, N., Munjal, R., \& Sharma, B. (2015). Genetic analysis of yield and heat stress related traits in wheat (Triticum aestivum L. em. Thell) using microsatellite markers. Journal of Applied and Natural Science, 7(2), 739-744.

11. Ilyas, N., Gull, R., Mazhar, R., Saeed, M., Kanwal, S., Shabir, S., \& Bibi, F. (2017). Influence of salicylic acid and jasmonic acid on wheat under drought stress. Communications in soil science and plant analysis, 48(22), 2715-2723.

12. Khan MIR, Khan NA (2013) Salicylic Acid and Jasmonates: Approaches in Abiotic Stress Tolerance. J Plant Biochem Physiol 1: el13.

13. Kim, Tae-Heon, Yeon-Jae Hur, Sang-Ik Han, Jun-Hyun Cho, Kyung-Min Kim, Jong-Hee Lee, You-Chun Song, Yeong-Up Kwon, and Dongjin Shin. 2017. "Drought-tolerant QTL qVDT11 leads to stable tiller formation under drought stress conditions in rice." Plant Science 256: 131-138.

14. Lydie Guilioni, Jacques Wery, Francois Tardieu. 1997. Heat Stress-induced Abortion of Buds and Flowers in Pea: Is Sensitivity Linked to Organ Age or to Relations between Reproductive Organs. Annals of Botany,80 (2): 159-168,

15. Lobell, D. B., Schlenker, W., and Costa-Roberts, J. (2011). Climate trends and global crop production since 1980. Science 333, 616-620. 
16. Lu, L., Liu, H., Wu, Y., \& Yan, G. (2020). Development and characterization of near-isogenic lines revealing candidate genes for a major 7AL QTL responsible for heat tolerance in wheat. Frontiers in Plant Science, 11, 1316.

17. Maulana, F., Ayalew, H., Anderson, J. D., Kumssa, T. T., Huang, W., \& Ma, X. F. (2018). Genome-wide association mapping of seedling heat tolerance in winter wheat. Frontiers in Plant Science, 9, 1272.

18. Mehmet Demir Kaya, Gamze Okçu, Mehmet Atak, Yakup Çıkıll, Özer Kolsarlcı .2006. Seed treatments to overcome salt and drought stress during germination in sunflower (Helianthus annuus L.) European Journal of Agronomy(24) 4:291-295

19. Ni, Z., Li, H., Zhao, Y., Peng, H., Hu, Z., Xin, M., \& Sun, Q. (2018). Genetic improvement of heat tolerance in wheat: recent progress in understanding the underlying molecular mechanisms. The Crop Journal, 6(1), 32-41.

20. Pang, Y., Chen, K., Wang, X., Xu, J., Ali, J., \& Li, Z. (2017). Recurrent selection breeding by dominant male sterility for multiple abiotic stresses tolerant rice cultivars. Euphytica, 213(12), 1-13.

21. Poudel, P. B., \& Poudel, M. R. (2020). Heat stress effects and tolerance in wheat: A review. Journal of Biology and Today's World, 9(3), 1-6.

22. Prince, S. J., Beena, R., Gomez, S. M., Senthivel, S., \& Babu, R. C. (2015). Mapping consistent rice (Oryza sativa L.) yield QTLs under drought stress in target rainfed environments. Rice, 8(1), 1-13.

23. Sabar, M., Shabir, G., Shah, S. M., Aslam, K., Naveed, S. A., \& Arif, M. (2019). Identification and mapping of QTLs associated with drought tolerance traits in rice by a cross between Super Basmati and IR55419-04. Breeding science, 69(1), 169-178.

24. Sadat, S., Saeid, K. A., Bihamta, M. R., Torabi, S., Salekdeh, S. G. H., \& Ayeneh, G. A. L. (2013). Marker assisted selection for heat tolerance in bread wheat. World applied sciences journal, 21(8), 1181-1189.

25. Shao, H. B., Chu, L. Y., Jaleel, C. A., Manivannan, P., Panneerselvam, R., and Shao, M. A. (2009). Understanding water deficit stress-induced changes in the basic metabolism of higher plants - biotechnologically and sustainably improving agriculture and the ecoenvironment in arid regions of the globe. Crit. Rev. Biotechnol. 29, 131-151.

26. Showler, Allan T. 2016. "Selected Abiotic and Biotic Environmental Stress Factors Affecting Two Economically Important Sugarcane Stalk Boring Pests in the United States" Agronomy 6, no. 1: 10

27. Singh, B., Mishra, S., Bohra, A., Joshi, R., \& Siddique, K. H. (2018). Crop phenomics for abiotic stress tolerance in crop plants. In Biochemical, physiological and molecular avenues for combating abiotic stress tolerance in plants (pp. 277-296). Academic Press.

28. Swamy, B. M., \& Kumar, A. (2013). Genomics-based precision breeding approaches to improve drought tolerance in rice. Biotechnology advances, 31(8), 1308-1318.

29. Reguera, M., Peleg, Z., \& Blumwald, E. (2012). Targeting metabolic pathways for genetic engineering abiotic stress-tolerance in crops. Biochimica et Biophysica Acta (BBA)-Gene Regulatory Mechanisms, 1819(2), 186-194.

30. Vikram, P., Kumar, A., Singh, A., \& Singh, N. K. (2012). Rice: Genomics-Assisted Breeding for Drought Tolerance. Improving Crop Resistance to Abiotic Stress, 715-731.

31. Wang, H., Wang, H., Shao, H., \& Tang, X. (2016). Recent advances in utilizing transcription factors to improve plant abiotic stress tolerance by transgenic technology. Frontiers in plant science, 7, 67.

32. Younis, Adnan, Fahad Ramzan, Yasir Ramzan, Faisal Zulfiqar, Muhammad Ahsan, and Ki B. Lim .2020. "Molecular Markers Improve Abiotic Stress Tolerance in Crops: A Review" Plants (9), no. 10: 1374. 
33. Zhang, Ke \& Congcong, Jin \& Wu, Lizhu \& Hou, Mingyu \& Dou, Shijuan \& Pan, Yanyun. (2014). Expression Analysis of a Stress-Related Phosphoinositide-Specific Phospholipase C Gene in Wheat (Triticum aestivum L.). PloS one. 9. e105061. 10.1371/journal.pone.0105061.

34. Zhang, X. Y., Liang, C., Wang, G. P., Luo, Y., \& Wang, W. (2010). The protection of wheat plasma membrane under cold stress by glycine betaine overproduction. Biologia Plantarum, 54(1), 83-88.

35. Zlatev, Z.; Lidon, F.C. An overview on drought induced changes in plant growth, water relations and photosynthesis. Emir. J. Food Agric. 2012, 24, 57-72.

36. SHRIVASTAVA, PRATEEK, et al. "Techno-Economic Evaluation of Tractor Operated Raised Bed Planters and Seed Drills for Cultivation of Wheat Crop." International Journal of Agricultural Science and Research, 7 (2), 349362 .

37. Mounika, M., K. U. Devi, and S. SUCHARITHA Devi. "Bioavailability of iron from foxtail millet and sorghum millet recipes." Int. J. Agric. Sci. Res.(IJASR) 7.4: 703-708.

38. Singh, P. K., et al. "Long term impact of tillage systems, irrigation and nitrogen on soil properties, growth, yield, nutrient uptake and quality of wheat (Triticum aestivum L.)." International Journal of Agricultural Research 7.4: 555-566.

39. Goyal, Megha, and Urmil Verma. "Wheat yield prediction using weather based statistical model in northern zone of Haryana." Int. J. Humanit. Soc. Sci 7.4: 47-50.

40. Ghumaan, GURDEEP KAUR, and PAWAN KUMAR Dhiman. "Perception of Fair Price Shop's Dealers Towards Public Distribution System." International Journal of Business and General Management 5.6: 11-24.

41. Singh, R. A. J. E. N. D. R. A. "Evaluation of nematicidal potency of botanical biopesticides in combination with triazophos against root knot nematode, Meloidogyne incognita infestation on chickpea, Cicer arietinum L." Int. J. App. \& Nat. Sci 4.3 : 75-82. 